\title{
Midterm results with thoracic endovascular aortic repair for chronic type $B$ aortic dissection with associated aneurysm
}

\author{
Cyrus J. Parsa, MD, Judson B. Williams, MD, Syamal D. Bhattacharya, MD, Walter G. Wolfe, MD, \\ Mani A. Daneshmand, MD, Richard L. McCann, MD, and G. Chad Hughes, MD
}

\begin{abstract}
Objective: Thoracic endovascular aortic repair for chronic type B aortic dissection with associated descending thoracic aneurysm remains controversial. Concerns include potential ischemic complications due to branch vessel origin from the chronic false lumen and continued retrograde false lumen/aneurysm sac pressurization via fenestrations distal to implanted endografts. The present study examines midterm results with thoracic endovascular aortic repair for chronic ( $>2$ weeks) type B aortic dissection with associated aneurysm to better understand the potential role of thoracic endovascular aortic repair for this condition.
\end{abstract}

\begin{abstract}
Methods: Between March 2005 and December 2009, 51 thoracic endovascular aortic repair procedures were performed at a single institution for management of chronic type B dissection. The indication for thoracic endovascular aortic repair was aneurysm in all cases. A subset of 7 patients (14\%) underwent placement of the EndoSure wireless pressure measurement system (CardioMEMS, Inc, Atlanta, Ga) in the false lumen adjacent to the primary tear for monitoring aneurysm sac/false lumen pulse pressure after thoracic endovascular aortic repair.
\end{abstract}

\begin{abstract}
Results: Mean patient age was $57 \pm 12$ years (range, $30-82$ years); 14 patients (28\%) were female. Mean aortic diameter was $6.2 \pm 1.4 \mathrm{~cm}$. There were no in-hospital/30-day deaths, strokes, or permanent paraplegia/paresis. There were no complications related to compromise of downstream branch vessels arising from the false lumen. Two patients (3.9\%) who had preexisting ascending aortic dilation had retrograde acute type A aortic dissection; both were repaired successfully. Median postoperative length of stay was 4 days. Mean follow-up is $27.0 \pm 16.5$ months (range, 2-60 months). Actuarial overall survival is $77.7 \%$ at 60 months with an actuarial aorta-specific survival of $98 \%$ over this same time period. Actuarial freedom from reintervention is $77.3 \%$ at 60 months. All patients with the EndoSure wireless pressure measurement system exhibited a decrease in aneurysm sac/false lumen pulse pressure indicating a depressurized false lumen. The aneurysm sac/false lumen pulse pressure ratio decreased from $52 \% \pm 27 \%$ at the predischarge measurement to $14 \% \pm 5 \%$ at the latest follow-up reading $(P=.029)$.
\end{abstract}

Conclusions: Thoracic endovascular aortic repair for chronic type B dissection with associated aneurysm is safe and effective at midterm follow-up. Aneurysm sac/false lumen pulse pressure measurements demonstrate a significant reduction in false lumen endotension, thus ruling out clinically significant persistent retrograde false lumen perfusion and provide proof of concept for a thoracic endovascular aortic repair-based approach. Longer-term follow-up is needed to determine the durability of thoracic endovascular aortic repair for this aortic pathology. (J Thorac Cardiovasc Surg 2011;141:322-7)

Approximately $80 \%$ to $85 \%$ of patients presenting with type B aortic dissection are managed successfully in the acute phase with medical therapy. ${ }^{1,2}$ Unfortunately, multiple studies ${ }^{3}$ have demonstrated that $20 \%$ to $40 \%$ of these patients will eventually require surgical intervention

From the Department of Surgery, Duke University Medical Center, Durham, NC. Disclosures: Authors have nothing to disclose with regard to commercial support. Read at the 36th Annual Meeting of The Western Thoracic Surgical Association, Ojai, California, June 23-26, 2010.

Received for publication June 26, 2010; revisions received Oct 11, 2010; accepted for publication Oct 24, 2010.

Address for reprints: G. Chad Hughes, MD, Director, Thoracic Aortic Surgery Program, Division of Thoracic and Cardiovascular Surgery, Department of Surgery, Box 3051, Duke University Medical Center, Durham, NC 27710 (E-mail: gchad. hughes@duke.edu).

0022-5223/\$36.00

Copyright (c) 2011 by The American Association for Thoracic Surgery

doi: $10.1016 /$ j.jtcvs.2010.10.043 in long-term follow-up, usually for aneurysmal degeneration of the chronically dissected aorta. Indications for surgical intervention generally include, in the absence of symptoms, a descending aortic diameter of $5.5 \mathrm{~cm}$ or more for fusiform aneurysms or $2 \mathrm{~cm}$ or more for saccular aneurysms or interval aortic growth of 5 to $10 \mathrm{~mm}$ or more per year. ${ }^{4}$ Some, however, have suggested a lower threshold based on data from E. Stanley Crawford's series ${ }^{5}$ demonstrating that $23 \%$ of ruptured chronic type B dissections occurred at an aortic diameter between 5 and $6 \mathrm{~cm}$, as well as data from the Mt Sinai group in which the median aortic diameter before rupture was $5.4 \mathrm{~cm} .{ }^{6}$ Lower thresholds also have been proposed for patients with connective tissue disorders (eg, Loeys-Dietz syndrome). ${ }^{3}$

Thoracic endovascular aortic repair (TEVAR) is a therapeutic advance for the management of acute complicated (rupture, malperfusion) type B aortic dissection, ${ }^{7}$ with 


\section{Abbreviations and Acronyms}

$\mathrm{CT}=$ computed tomography

TEVAR $=$ thoracic endovascular aortic repair

significantly reduced rates of morbidity and mortality compared with contemporary open repair data. ${ }^{2}$ However, controversy exists with regard to the use of TEVAR for the management of chronic type B dissection with aneurysm. ${ }^{8}$ Critics have cited the fact that the intimal flap becomes thickened in chronic dissections and does not immediately reapproximate to the native aortic wall, ${ }^{9}$ as well as the fact that distal fenestrations between the true and false lumens become well established over time such that complete or near-complete false lumen thrombosis is less likely in the chronic setting. ${ }^{4}$ For these reasons, the Society of Thoracic Surgeons Endovascular Surgery Task Force ${ }^{4}$ gave TEVAR for chronic type B dissection a class IIb (efficacy not established) evidence rating. Thus, the purpose of the present study was to examine midterm results with TEVAR for chronic ( $>2$ weeks) type B aortic dissection with associated aneurysm to better understand the potential role of TEVAR for this condition.

\section{PATIENTS AND METHODS}

Between March 23, 2005 (date of Food and Drug Administration approval of the first available thoracic device in the United States), and December 31, 2009, 274 TEVAR procedures were performed at a tertiary referral university hospital for the treatment of thoracic aortic pathology. Of these, $51(18.6 \%)$ were for management of chronic dissection of the descending thoracic aorta and form the basis of this report. The indication for intervention in all cases was aneurysmal degeneration of the chronically dissected aorta. In all patients, the aneurysmal segment was limited to the descending thoracic aorta, generally immediately adjacent to the primary tear, with aortic diameters amenable to distal endograft seal at the level of the celiac axis. Criteria for repair included rapid enlargement $(>5 \mathrm{~mm}$ in 6 months $)(\mathrm{n}=7)$ or absolute size. For fusiform aneurysms, this included a diameter of $5.5 \mathrm{~cm}$ or more $(\mathrm{n}=41)$, whereas for saccular aneurysms a protrusion of $2 \mathrm{~cm}$ or more beyond the aortic wall for the saccular component was considered an indication for treatment in the absence of symptoms $(\mathrm{n}=3) .{ }^{4}$ The study was approved by the Duke Institutional Review Board, and the institutional review board waived the need for individual patient consent.

Devices used were Gore TAG (WL Gore \& Associates, Flagstaff, Ariz) $(\mathrm{n}=36 ; 71 \%)$, Zenith TX2 (Cook Medical Incorporated, Bloomington, Ind) $(\mathrm{n}=12 ; 24 \%)$, and Medtronic Talent (Medtronic Inc, Santa Rosa, Calif) $(\mathrm{n}=1 ; 2 \%) ; 2$ patients $(4 \%)$ received more than 1 type of device. Five patients $(10 \%)$ required an iliac or infrarenal aortic conduit to allow safe introduction of the introducer sheath necessary for the procedure. The left subclavian artery was partially or fully covered in 35 patients $(69 \%)$, of whom $6(17 \%$ of those in whom the left subclavian was covered) underwent adjunctive left carotid-subclavian bypass during the same operation as endovascular repair. Indications for left carotid-subclavian bypass were as previously described. ${ }^{10}$

To definitively evaluate the hypothesis that continued retrograde false lumen/aneurysm sac pressurization via fenestrations distal to implanted endografts occurs after TEVAR, ${ }^{4} 7$ patients $(14 \%)$ underwent placement of an EndoSure wireless pressure measurement system (CardioMEMS,
Inc, Atlanta, Ga) at the time of TEVAR as previously described. ${ }^{11}$ The EndoSure device was intentionally positioned within the false lumen/aneurysm sac adjacent to the primary tear via an existing distal reentry tear that enabled measurement of potential false lumen pressurization (Figure 1). The mechanism of the EndoSure sensor has been described. ${ }^{11}$ Briefly, alterations in surrounding false lumen/aneurysm sac pressure change the capacitance and therefore the resonant frequency of the sensor. An external antenna activates the sensor via a radiofrequency impulse and then receives the pressure-dependent change via the resonant frequency of the sensor, which is converted into a real-time pressure measurement.

Preoperative planning of endograft procedures was performed using the TeraRecon system (TeraRecon Inc, San Mateo, Calif), which allows highly accurate centerline measurements of flow lumen diameter to assess landing zones and iliofemoral access vessels. Intravascular ultrasound (Volcano Corporation, San Diego, Calif) was used in all cases to verify true lumen access, identify the primary entry tear and areas of distal fenestration, and evaluate proximal and distal landing zones as previously described. ${ }^{8}$

All endovascular procedures were performed in the operating room under general anesthesia; adjunctive transesophageal echocardiography was used routinely. On-line monitoring of spinal cord function with somatosensory and motor evoked potentials was used intraoperatively in elective cases and when available for urgent/emergency cases $(n=43$ cases monitored; $84 \%$ ) using previously described techniques. ${ }^{12}$ Cerebrospinal fluid drainage was used selectively $(n=9 ; 18 \%)$ for previously described indications. $^{10}$

Comorbidities were defined using standard definitions. All procedural outcomes and complications were prospectively recorded. Patient follow-up included clinical examination, 4-view chest $\mathrm{x}$-ray, and computed tomography (CT) angiography at 1, 6, and 12 months postoperatively and annually thereafter. In addition, 3-month follow-up assessment and imaging were obtained in patients with an endoleak identified at 1 month if the decision for initial endoleak observation was made. False lumen/aneurysm sac pressure tracings were acquired intraoperatively pre- and postexclusion of the false lumen, at the time of discharge, and at each follow-up visit. All follow-up was done at the Duke University Center for Aortic Surgery. This report includes all data collected through the patients' most recent follow-up visit. In addition, the social security death index was queried (http://ssdi.rootsweb.com/) to confirm all patient deaths. For those patients dying in follow-up, cause of death was confirmed by review of medical records or family interview in all cases.

Data were warehoused in an encrypted spreadsheet (Excel 2007, Microsoft, Redmond, WA). Descriptive and summary statistics with mean and standard deviation were calculated. Continuous variables were compared using the 2-tailed paired Student $t$ test. Statistical analyses were performed using SAS version 9.13 SP4 (SAS Institute Inc, Cary, NC). Survival analyses were performed using the Kaplan-Meier method. All data are presented in accordance with the "Reporting standards for endovascular aortic aneurysm repair" of the Ad Hoc Committee for Standardized Reporting Practices in Vascular Surgery of The Society for Vascular Surgery/American Association for Vascular Surgery. ${ }^{13}$

\section{RESULTS \\ Patient Demographics}

Mean aortic diameter was $6.2 \pm 1.4 \mathrm{~cm}$. All patients had a patent false lumen at the time of TEVAR, and the ratio of true lumen to total aortic caliber was $34 \% \pm 15 \%$. The interval from initial dissection event to stent graft repair was a mean of $46.2 \pm 53.7$ months (range, 2-216 months). Patient demographics are presented in Table 1. Approximately half the cohort $(n=24 ; 47 \%)$ had prior aortic surgery, including open abdominal aortic aneurysm repair $(\mathrm{n}=3$; $13 \%)$, endovascular aortic repair $(n=2 ; 8 \%)$, open 

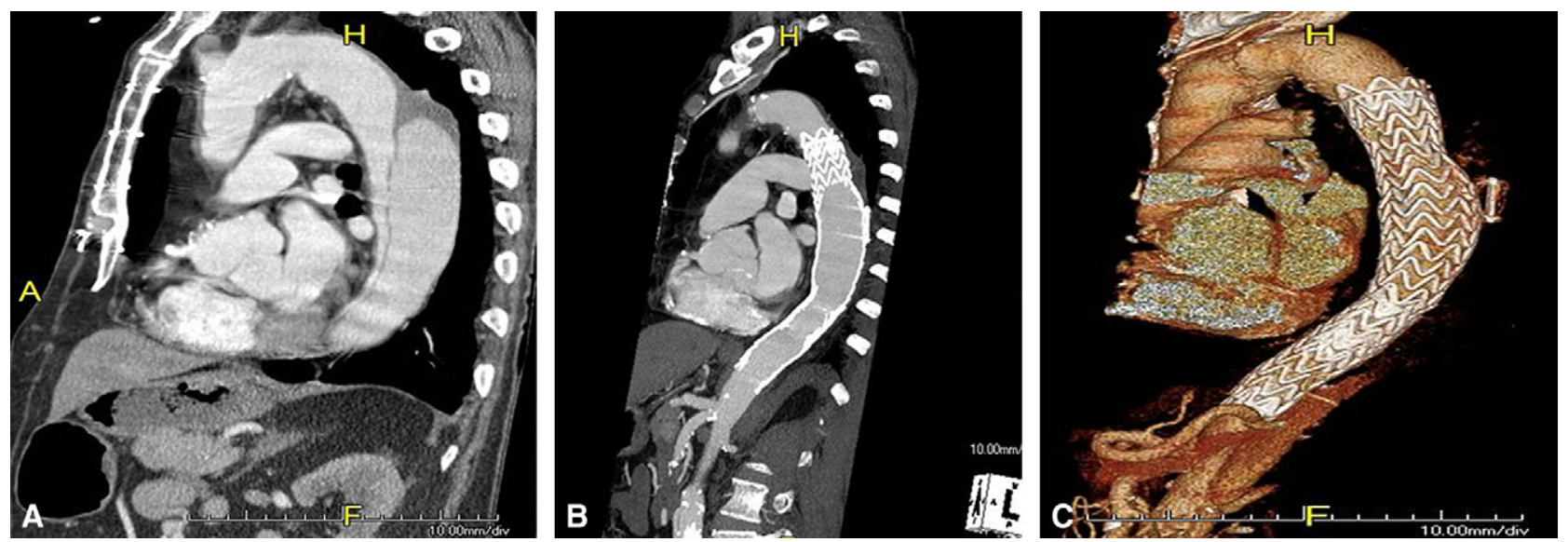

FIGURE 1. Mid-sagittal CT angiogram images of (A) thoracic aorta with chronic type B dissection with aneurysmal dilatation of the false lumen of the chronically dissected aorta and (B) after TEVAR with false lumen EndoSure (CardioMEMS, Inc, Atlanta, Ga) aneurysm sac/false lumen pulse pressure sensor in place. True lumen is reestablished with thrombosed false lumen and aneurysm sac/false lumen pulse pressure device. C, Three-dimensional reconstruction of B.

descending thoracic aortic repair $(\mathrm{n}=5 ; 21 \%)$, or prior type A dissection or arch repair $(n=14 ; 58 \%)$. Extent of aortic coverage by the endograft(s) is presented in Table 2. Thirteen cases $(25 \%)$ were urgent $(\mathrm{n}=12$ [24\%] due to acutely symptomatic aneurysms) or emergency $(n=1[2 \%]$ due to hemoptysis).

\section{Procedural (30-Day) Outcomes}

The thoracic endografts were deployed in the intended position in all patients. The median number of stent graft components per case was $2.0 \pm 0.7$ (range 1-4); the range of device diameters used was 26 to $46 \mathrm{~mm}$. Thirty-day/inhospital rates of death, stroke, and permanent paraplegia/ paresis were all $0 \%$. Renal failure with new dialysis occurred in $2.0 \%$ of patients $(n=1)$. Two patients $(3.9 \%)$ who had preexisting ascending aortic dilation, 1 of whom had a congenital bicuspid aortic valve, had retrograde acute type A aortic dissection after implantation of a Zenith TX2 device, which has proximal barbs for fixation. The first occurred intraoperatively and was detected via completion intravascular ultrasound and transesophageal echocardiography; immediate repair with mechanical valved conduit (patient with congenital bicuspid aortic valve) and hemi-

TABLE 1. Patient demographics

\begin{tabular}{lc}
\hline Median age & $57 \pm 12$ y (range, 30-82) \\
Female & $\mathrm{n}=14(28 \%)$ \\
Hypertension & $\mathrm{n}=48(94 \%)$ \\
Active or recent smoker & $\mathrm{n}=27(53 \%)$ \\
Diabetes & $\mathrm{n}=4(8 \%)$ \\
History of myocardial infarction & $\mathrm{n}=2(4 \%)$ \\
History of stroke & $\mathrm{n}=3(6 \%)$ \\
COPD & $\mathrm{n}=9(18 \%)$ \\
Chronic renal insufficiency $\quad \mathrm{n}=12(24 \%)$ \\
$\quad$ baseline creatinine $\geq 1.5)$ & \\
COPD, Chronic obstructive pulmonary disorder. &
\end{tabular}

arch replacement was performed, and the patient did well. The second was delayed, occurring on postoperative day 5 when new-onset chest pain developed in the patient, diagnosed by CT angiography (Figure 2). This was likewise an emergency repair via valve resuspension with ascending aorta and hemi-arch replacement with good result. Median postoperative length of stay was 4 days (25th, 75th percentile $=2,6$ days, respectively).

\section{Follow-up Outcomes}

Follow-up is $100 \%$ complete. Mean duration of followup is $27.0 \pm 16.5$ months (range, 2-60). Overall actuarial survival is $77.7 \%$ at 60 months, with an aorta-specific actuarial survival of $98 \%$ during this same time interval (Figure 3). Of the late deaths, $1(2.0 \%)$ was aortic-related and the remainder $(n=9)$ were due to comorbid conditions. The single aortic-related death was a patient who underwent emergency TEVAR for a symptomatic (pain and hemoptysis) 9.3-cm mycotic (Salmonella) descending aneurysm secondary to chronic type B dissection. She reported persistent dysphagia 1.5 months after endograft placement, and workup indicated the presence of an aortoesophageal fistula with the endograft visible on upper endoscopy. She subsequently underwent conversion to open repair and died of complications related to open repair after 30 days.

The previously patent false lumen/aneurysm sac adjacent to the primary tear was thrombosed in all patients in the series after TEVAR. Overall aortic diameter decreased from $6.2 \pm 1.4 \mathrm{~cm}$ preoperatively to $5.0 \pm 1.4 \mathrm{~cm}$ at latest follow-up $(P=.0002)$. Further, $87 \%$ of patients with imaging follow-up beyond 6 months demonstrate complete reverse remodeling of the thoracic aorta in the region of prior aneurysm (Figure 4). The incidence of type I endoleak at any follow-up visit was $7.8 \%(n=4)$; all were seen on the initial postoperative CT scan and treated successfully with 
TABLE 2. Length of aortic coverage by the endograft(s)

\begin{tabular}{lc}
\hline \multicolumn{1}{c}{ Extent of aortic "pavement" } & No. of patients (\%) \\
\hline Left subclavian artery to T6 & $19(37.3 \%)$ \\
Mid thoracic aorta to celiac axis & $0(0 \%)$ \\
Left subclavian artery to celiac axis & $32(62.7 \%)$ \\
\hline
\end{tabular}

additional endovascular repair at 1 week and 2, 5, and 6 months postoperatively; no type I endoleaks were present at most recent follow-up. There were no type III endoleaks noted in the study cohort. Two patients $(3.9 \%)$ underwent successful endovascular occlusion of type II endoleaks caused by retrograde flow from the left subclavian artery, both at 3 months postoperatively. Three patients $(5.9 \%)$ required additional distal endografting for metachronous downstream aortic dilation; reintervention was successful in all cases. Two patients $(3.9 \%)$ required late conversion to open repair, the aforementioned conversion for aortoesophageal fistula and another for continued sac expansion caused by retrograde filling of the distal false lumen from a large reentry tear in the visceral segment. Actuarial freedom from reintervention was $77.3 \%$ at 60 months post-TEVAR (Figure 5).

In the subset of patients $(\mathrm{n}=7 ; 14 \%)$ undergoing EndoSure placement in the false lumen, all exhibited a decrease in aneurysm sac/false lumen pulse pressure, although 1 patient later manifested an increase to near-systemic pressures at the 6-month reading because of sensor "sandwiching" between the stent graft and outer aortic false lumen wall caused by significant reverse aortic remodeling. Of the re-

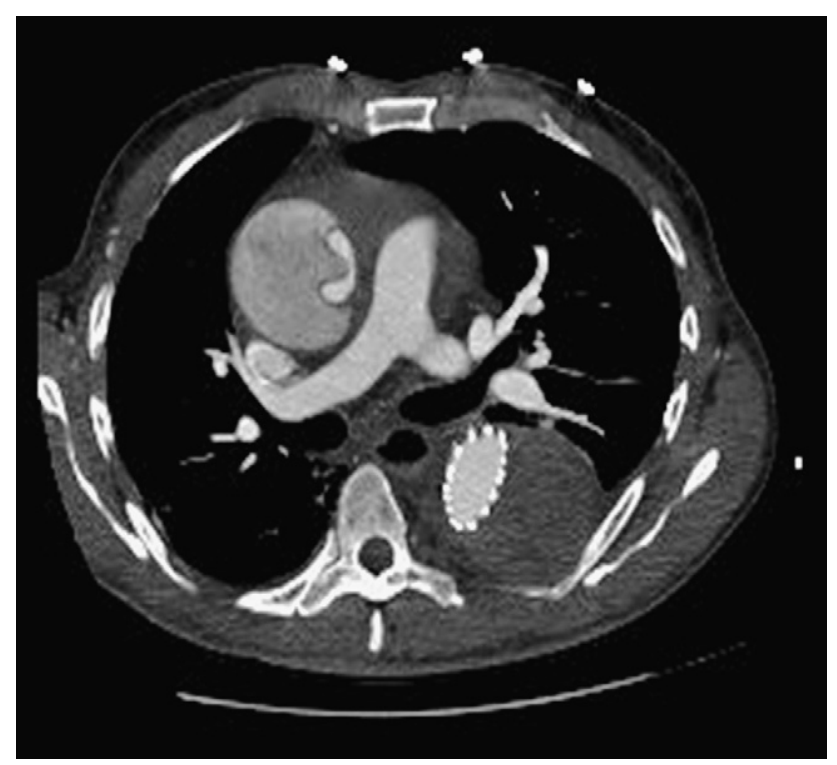

FIGURE 2. CT angiogram demonstrating acute type A aortic dissection 5 days after TEVAR for a chronic type B dissection with associated aneurysm. Note Zenith TX2 stent graft in descending thoracic aorta with completely thrombosed descending false lumen/aneurysm sac and new dissection flap in dilated ascending aorta.

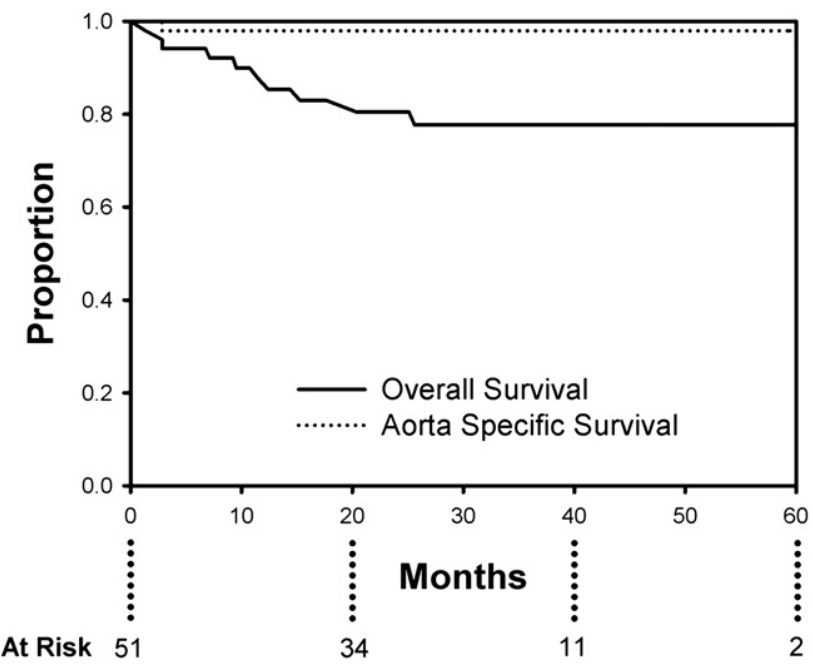

FIGURE 3. Actuarial (Kaplan-Meier) overall (solid line) and aortaspecific (dotted line) survival at 60 months post-endovascular repair for chronic dissection with aneurysm. Overall survival is $77.7 \%$ and aortaspecific survival is $98 \%$ at 60 months.

maining 6 patients, the aneurysm sac/false lumen pulse pressure ratio (ratio of pulse pressure in the false lumen/aneurysm sac to systemic pulse pressure) decreased from $52 \%$ $\pm 27 \%$ at the predischarge measurement to $14 \% \pm 5 \%$ at the latest follow-up reading $(P=.029)$. The difference of the means equals a $38 \%(95 \%$ confidence interval, 19-70.4) further reduction in false lumen/aneurysm sac pressure during follow-up. Similarly, the reduction in aortic diameter in this subset was statistically significant, with maximal aortic diameter decreasing from $5.6 \pm 0.8 \mathrm{~cm}$ preoperatively to $4.6 \pm 0.9 \mathrm{~cm}$ at latest follow-up $(P=.0024)$.

\section{DISCUSSION}

Controversy persists regarding application of TEVAR for the management of chronic type B dissection with aneurysm $^{8}$ mainly because of concerns regarding the thickened intimal flap that does not readily reapproximate to the native aortic wall, ${ }^{9}$ as it does after TEVAR for acute dissection, as well as the fact that distal fenestrations between the true and false lumens become more established over time such that complete or near-complete false lumen thrombosis may be less likely in the chronic setting. ${ }^{4}$ For these reasons, the Society of Thoracic Surgeons Endovascular Surgery Task Force ${ }^{4}$ gave TEVAR for chronic type B dissection a class IIb (efficacy not established) evidence rating.

The present study addresses some of these concerns. First, overall aortic diameter decreased significantly over the follow-up period consistent with successful and sustained exclusion of the chronic false lumen/aneurysm sac. Second, despite the presumed persistence of fenestrations distal to the endografts, retrograde pressurization of the false lumen does not occur as evidenced by false lumen/ aneurysm sac pressures that were approximately $14 \%$ of 


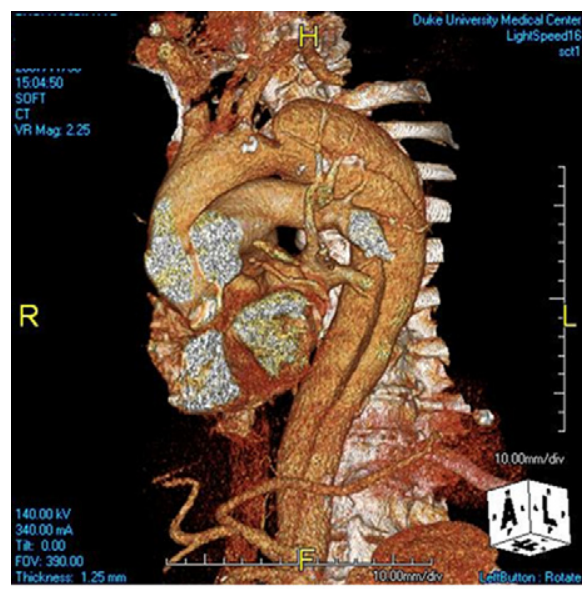

A

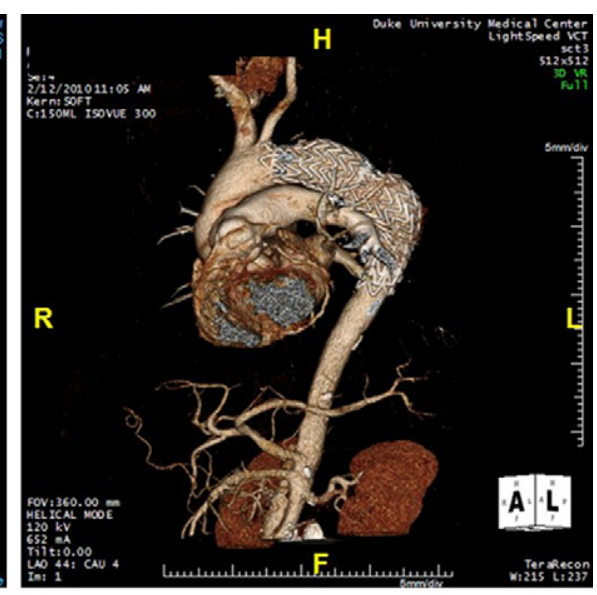

B

FIGURE 4. Preoperative (A) and 2-year post-TEVAR follow-up (B) 3-dimensional CT angiograms of a chronic type B dissection with associated aneurysm. Note large aneurysm with chronic false lumen in (A) with complete reverse remodeling of entire thoracic aorta in (B) such that no residual aneurysm/ dissection remains in the thoracic aorta.

systemic pressures at latest follow-up. There was a single patient early in the series who required late open conversion for continued false lumen/sac enlargement caused by retrograde flow from a large visceral segment reentry tear. However, our experience in treating this disease with TEVAR has led us to believe that significant retrograde false lumen pressurization via distal fenestrations to an extent capable of promoting continued sac expansion does not occur in the absence of an outflow route, which is typically the left subclavian artery. Two patients in this series underwent late (3 months) endovascular occlusion procedures for type II endoleaks from the left subclavian artery with continued false lumen/aneurysm sac perfusion. In reality, however, these were not true endoleaks because the flow in the subclavian was actually retrograde away from the false lumen

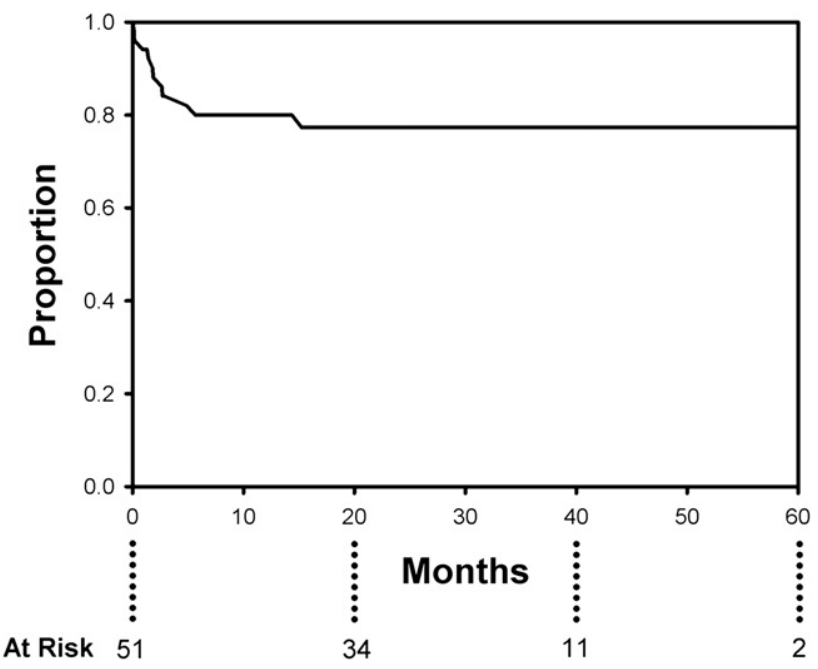

FIGURE 5. Actuarial (Kaplan-Meier) freedom from reintervention is $80 \%$ at 12 months and $77.3 \%$ at 60 months post-endovascular repair. potentiated by inflow from downstream fenestrations between the true and false lumens. In both patients, the false lumen/aneurysm sac thrombosed and then began to decrease in size after the subclavian was occluded. As in 1 of these 2 patients, this retrograde false lumen flow may be missed if the timing of intravenous contrast and CT image acquisition does not permit adequate opacification of the false lumen; acquisition of delayed CT images should be mandatory during follow-up imaging to ensure detection of continued false lumen flow. In retrospect, we suspect this to be the case with the earlier patient in this series requiring open conversion, because his left subclavian artery remains patent on follow-up imaging after his open conversion despite presumed left subclavian artery coverage at the index endograft repair. Finally, consistent with these low false lumen/sac pressures is the finding of significant reverse aortic remodeling after depressurization of the chronic false lumen/aneurysm sac. This reverse remodeling suggests that, despite the thickened nature of the chronic dissection flap ( $3.1 \pm 1.8 \mathrm{~mm}$ in this series), the flap is able to reapproximate to the outer aortic wall with time, generally at 6 months or more follow-up in the present series. Overall, the findings suggest "proof of concept" for TEVAR for the treatment of chronic descending dissection with associated aneurysm. Similar results for TEVAR in chronic descending dissection with aneurysm have been reported by the Arizona Heart Institute. ${ }^{14}$

Benchmark data for open repair of chronic type B dissection in a cohort of $n=104$ patients were recently published by the Mt Sinai group. ${ }^{15}$ The demographics of patients in that study were similar to the current report with nearly identical mean age and similar comorbidities. In contrast with the current report with no 30-day deaths, strokes, or spinal cord ischemic events, however, they reported $10 \%$ 30 day/in-hospital mortality, $6 \%$ stroke, and 5\% paraplegia 
with open repair. Further, the tracheostomy rate was $14 \%$ with a median postoperative length of stay of 13 days versus $0 \%$ and 4 days, respectively, in the current study. Admittedly, however, the extent of aortic disease in the Mt Sinai study was greater than the current report with $16 \%$ of patients requiring visceral reimplantation because of thoracoabdominal aneurysmal involvement. Regardless, the current report suggests reduced rates of morbidity and mortality with TEVAR for chronic type B dissection with aneurysm compared with contemporary open repair data, similar to findings with acute complicated (rupture, malperfusion) type B aortic dissection. ${ }^{7}$ Although follow-up is midterm at present, the current study also reports an improved overall survival at 5 years $(78 \%$ current study vs $68 \%$ Mt Sinai series ${ }^{15}$ ) after TEVAR versus open repair for chronic descending dissection. Because the extent of aortic disease was somewhat greater in the Mt Sinai series, however, we consider this observation as a preliminary trend with further study required.

The results of the current study highlight an increased need for reintervention with a TEVAR-based approach compared with open repair, although the need for reoperation after open repair increases significantly with duration of follow-up and was $17 \%$ at 10 years in the Mt Sinai series. ${ }^{15}$ In the present series, the majority of reinterventions used endovascular techniques with minimal morbidity and mortality. Notably, the current data suggest reintervention is more likely in the first year after TEVAR with the incidence decreasing significantly over the remainder of the study period (Figure 5).

Our approach is to individualize the use of open repair or TEVAR based on patient anatomy and comorbid characteristics. Open repair is clearly the preferred option in patients with connective tissue disorders, such as Marfan or LoeysDietz syndrome given the poor results of TEVAR in this setting $^{16}$ and the fact that these patients are typically young and do well with open repair. ${ }^{17,18}$ Also, patients with thoracoabdominal aneurysm will not be treatable with a TEVAR approach, although "hybrid" repair may be preferable in some based on comorbid factors. ${ }^{19}$ Finally, younger patients with chronic dissection secondary to malignant hypertension may also be better served with open repair, although this is not supported by data at present and the decision regarding TEVAR versus open repair should ideally be made by a surgical team with expertise in both techniques, and consideration of institutional results with each technique should factor heavily into the decisionmaking process.

\section{CONCLUSIONS}

The current results, consistent with other series published over the last year, ${ }^{14}$ suggest a potential benefit for endovascular therapy in chronic descending dissection complicated by aneurysmal degeneration. Limitations of this study include its retrospective nature and reliance on singleinstitution data. Further, the duration of follow-up clearly needs to increase to assess the durability of this therapeutic modality. By drawing on parallel results from other series and longer-term data currently available for other TEVAR indications (eg, aneurysmal disease and acute complicated type B dissections), it does seem that this technology augments the surgical armamentarium for the treatment of chronic descending thoracic aortic dissection.

The authors thank Betty C. Tong, MD, MHS, for statistical review of this article.

\section{References}

1. Trimarchi S, Nienaber CA, Rampoldi V, Myrmel T, Suzuki T, Bossone E, et al Role and results of surgery in acute type B aortic dissection. Insights from the International Registry of Acute Aortic Dissection (IRAD). Circulation. 2006; 114:I-357-64

2. Tsai TT, Fattori R, Trimarchi S, Isselbacher E, Myrmel T, Evangelista A, et al. Long-term survival in patients presenting with type B acute aortic dissection. Insights from the International Registry of Acute Aortic Dissection. Circulation 2006; 114:2226-31

3. Parsa CJ, Hughes GC. Surgical options to contend with thoracic aortic pathology. Semin Roentgenol. 2009;44:29-51.

4. Svensson LG, Kouchoukos NT, Miller DC, Bavaria JE, Coselli JS, Curi MA, et al. Expert consensus document on the treatment of descending thoracic aortic disease using endovascular stent-grafts. Ann Thorac Surg. 2008;85:S1-41.

5. Crawford ES. The diagnosis and management of aortic dissection. JAMA. 1990; 264:2537-41.

6. Lansman SL, Hagl C, Fink D, Galla JD, Spielvogel D, Ergin MA, et al. Acute type B aortic dissection: surgical therapy. Ann Thorac Surg. 2002;74:1833-5; discussion 1857-63.

7. Parker JD, Golledge J. Outcome of endovascular treatment of acute type B aortic dissection. Ann Thorac Surg. 2008;86:1707-12.

8. Parsa CJ, Schroder JN, Daneshmand MA, McCann RL, Hughes GC. Midterm results for endovascular repair of complicated acute and chronic type B aortic dissection. Ann Thorac Surg. 2010;89:97-102.

9. Kasirajan K, Milner R, Chaikof EL. Late complications of thoracic endografts. J Vasc Surg. 2006;43:94-99A.

10. Hughes GC, Daneshmand MA, Swaminathan M, Nienaber JJ, Bush EL, Husain AH, et al. "Real World" thoracic endografting: results with the Gore TAG device 2 years following U.S. FDA approval. Ann Thorac Surg. 2008;86:1530-8.

11. Parsa CJ, Daneshmand MA, Lima B, Balsara K, McCann RL, Hughes GC. Utility of remote wireless pressure sensing for endovascular leak detection after endovascular thoracic aneurysm repair. Ann Thorac Surg. 2010;89:446-52.

12. Husain AH, Swaminathan M, McCann RL, Hughes GC. Neurophysiologic intraoperative monitoring during endovascular stent graft repair of the descending thoracic aorta. J Clin Neurophysiol. 2007;4:328-35.

13. Chaikof EL, Blankensteijn JD, Harris PL, White GH, Zarins CK, Bernhard VM, et al. Reporting standards for endovascular aortic aneurysm repair. J Vasc Surg. 2002;35:1048-60.

14. Rodriguez JA, Olsen DM, Lucas L, Wheatley G, Ramaiah V, Diethrich EB. Aortic remodeling after endografting of thoracoabdominal aortic dissection. $J$ Vasc Surg. 2008;47:1188-94

15. Zoli S, Etz CD, Roder F, Mueller CS, Brenner RM, Bodian CA, et al. Long-term survival after open repair of chronic distal aortic dissection. Ann Thorac Surg. 2010;89:1458-66.

16. Cooper DG, Walsh SR, Sadat U, Hayes PD, Boyle JR. Treating the thoracic aorta in Marfan syndrome: surgery or TEVAR? J Endovasc Ther. 2009;16:60-70.

17. Rajagopal K, Rogers JG, Lodge AJ, Gaca JG, McCann RL, Milano CA, et al. Two-stage total cardioaortic replacement for end-stage heart and aortic disease in Marfan syndrome: case report and review of the literature. J Heart Lung Transplant. 2009;28:958-63.

18. Williams MW, Wechsler SB, Hughes GC. Two-stage total aortic replacement for Loeys-Dietz syndrome. J Cardiac Surg. 2010;25:223-4.

19. Hughes GC, McCann RL. Hybrid thoracoabdominal aortic aneurysm repair: Concomitant visceral revascularization and endovascular aneurysm exclusion Semin Thorac Cardiovasc Surg. 2009;21:355-62. 